\title{
VICTORY - A 3D Search Engine over P2P and wireless P2P Networks
}

Petros Daras
Centre for Research and Technology
Hellas - Informatics and Telematics
Institute
Thessaloniki, Greece
+302310464160
daras@iti.gr
Jernej Trnkoczy
University of Ljubljana
Ljubljana, Slovenia
+38614768853
jernej.trnkoczy @ldos.fe.uni-lj.si
Ralph Traphoener
Empolis GmbH
Kaiserslautern, Germany
+4952418074377

\author{
Dimitrios Tzovaras \\ Centre for Research and Technology \\ Hellas - Informatics and Telematics \\ Institute \\ Thessaloniki, Greece \\ +302310464160 \\ tzovaras@iti.gr
Andrea Sanna Politecnico Di Torino, Italy $+390115647027$ \\ andrea.sanna@polito.it
}

Jasmin Franz

Empolis $\mathrm{GmbH}$

Kaiserslautern, Germany

+ 4952418074377

jasmin.franz@empolis.com

\section{c.malavazos@hypertech.gr}

Konstantina Geramani

TWT GmbH

Stuttgart, Germany

+490758171510

konstantina.geramani@twt-

$$
\text { gmbh.de }
$$

$$
\begin{gathered}
\text { Nikiforos Ploskas } \\
\text { Teletel S.A. } \\
\text { Athens, Greece } \\
\text { +30 } 2106983393 \\
\text { N.Ploskas@teletel.eu }
\end{gathered}
$$

Gerd-Joachim Wintterle

ALCATEL-LUCENT Deutschland AG

Stuttgart, Germany

+4971182144367

J.Wintterle@alcatel-lucent.de

\author{
Stefan Dobravec \\ University of Ljubljana \\ Ljubljana, Slovenia \\ +38614768 853
}

stefand@ldos.fe.uni-li.si
Gianluca Paravati
Politecnico Di Torino, Italy + 390115647027

gianluca.paravati@polito.it

Timotheos Kastrinogiannis

Hypertech S.A

Athens, Greece

+302106996157

t.kastrinogiannis@hypertech.gr

\author{
Mathias Gumz \\ Living Solids $\mathrm{GmbH}$ \\ Magdeburg, Germany \\ +49391251920
}

mathias.gumz@livingsolids.de

\footnotetext{
ABSTRACT

Our information society is being overflowed with digital data of various kinds. In order for the users to find relevant information, novel digital repositories should provide search mechanisms that
} 
are both content and context based, facilitating the formulation of queries and enabling search by example using 3D/2D objects, sketches, etc. At the same time the repositories should scale to the growing number of users. This paper presents the main achievements of the IST project VICTORY that aims at developing an innovative distributed digital repository introducing novel search, retrieval, and visualization capabilities to a desktop and mobile P2P networks.

\section{Keywords}

3D search engine, peer-to-peer networks, mobile terminals, QoE

\section{INTRODUCTION}

Three-dimensional (3D) content retrieval is overlooked in most commercial search engines, while at the same time it is expected to represent a huge amount of data stored in the Internet. Indeed, "geometry is poised to become the fourth wave of digitalmultimedia communication", where "the first three waves were sound in the 1970s, images in the '80s, and video in the '90s" [1]. With the wide availability of digital media capturing devices, over the last decade, it is now possible to acquire $3 \mathrm{D}$ data of a physical object in a few seconds and produce a digital model of its geometry that can be easily shared on the Internet.

The case of content/context based search where a user searches for a specific or similar objects (e.g. a photo of a flower in a vase placed on the table) is currently a hot research topic. In that case, the user visualizes the object which consists of all the information $\mathrm{s} / \mathrm{he}$ wants to describe and which is derived from the experience of seeing that object. Thus, a digital repository that provides both content and context based search better suits to the user's needs. While it is easier to develop such a digital repository as a centralized standalone solution, a distributed P2P (Peer-toPeer) based option would solve storage space, network bandwidth and processing power related problems of a centralized approach. This is especially critical for systems that support a large community of users which are allowed to add their own digital content and thus enrich the repository.

Further, in recent years the mobile handheld devices (e.g. mobile phones and PDAs) have developed to such extent that they are often used as web terminals. However, they are still underpowered in terms of processing power, rendering capabilities and network connection when compared to a PC. Nevertheless, wide adoption of these devices calls for their integration in the proposed search and retrieval framework. Therefore, mechanisms able to utilize not only the distributed data storage, but also the computational power of entities in the network need to be developed.

The IST VICTORY project (Audio-VIsual ConTent search retrieval in a distributed $\mathrm{P} 2 \mathrm{P}$ repositORY) [2], a project which started in January 2007, aims to tackle the mentioned problems. Its main purpose is to build a distributed repository of heterogeneous visual objects, accompanied with related textual documents, videos and images, that we call MultiPedia objects and a search engine able of retrieving $3 \mathrm{D}$ objects. This paper describes the project and its main achievements so far paying specific attention on the architectural aspects of the repository, the hybrid network and on the innovative applications that have already been developed.
The paper is organized as follows: Section 2 presents the Victory architecture while Section 3 basically reviews technologies and methodologies used within the project. Finally, conclusions are drawn in Section 4.

\section{THE VICTORY ARCHITECTURE}

The main elements of the VICTORY network architecture are depicted in Figure 1 and include:

Edge Peers are interconnected desktop computers and mobile devices. Edge peers provide share and download services, therefore allowing for other peers to download physical files that are distributed in the P2P network. Storage of the physical data files on edge peers eliminates the enormous storage space and network bandwidth requirements that would arise in case of a completely centralized server-based solution and offers the user a sense of security since his/her property remains on his/her PC. Edge peers are responsible also for local low-level feature extraction from the shared 3D objects, and in this way they contribute their processing power to the system. The set of functionalities for mobile and desktop peers naturally differs because of different processing and storage capabilities they posses.

Super Peers are in the first place providing search services of distributed MultiPedia repository. Each of super peers contains a part of the distributed search engine index that is used to map search queries to search results. Another important functionality of Super peers is acting as a rendezvous and relay, allowing for resolving edge peers' endpoint addresses and providing mechanisms for firewall and NAT traversal. Super peers are also responsible for hosting other VICTORY services. Some of the super peers (rendering providers) will enable the remote visualization of complex 3D geometries that cannot be processed on peers with low processing capabilities. Super Peers encompass also a set of dedicated servers that will provide specialized services like QoE (Quality of Experience) coordination services and identity provision service. Super peers also function as gateways for mobile devices. They act as a bridge between mobile clients, which are not capable of using $\mathrm{P} 2 \mathrm{P}$ communication mechanisms, and the rest of the system. The gateway also performs various tasks on behalf of the mobile users, where the processing power and bandwidth of the mobile device limits the set of functionalities.

As can be seen, a hybrid between pure P2P and Grid-like client server approach was selected for the VICTORY project. The classical P2P mechanisms are mainly used to support sharing and downloading services which are running also on devices that are hidden behind firewalls and NATs. These mechanisms provide the downloading of distributed data files from many peers simultaneously. VICTORY uses JXTA [3] technology for establishing an overlay P2P network over an existing low-level networking infrastructure. All other services (rendering, QoE coordination, identity management, etc.) are located on machines with public and static IP addresses, therefore a Grid-like client server approach is used to access them. 
This hybrid architecture was selected because it offers the following advantages:

The advanced low-level features-based search mechanism is extremely complex. Running processes of this complexity on every peer in the network is not a viable option. Instead a set of servers providing this capability is a better solution.

The need to control performance and reliability dictates a partially centralized solution.

Strict security needs in professional business applications can be better managed with this kind of architecture.

The efficiency of search (better precision and recall measures) can be improved by grouping peers with the same interests into groups. The easiest way of doing this is connecting peers with the same interest on a dedicated super peer and then querying for results only within certain group.

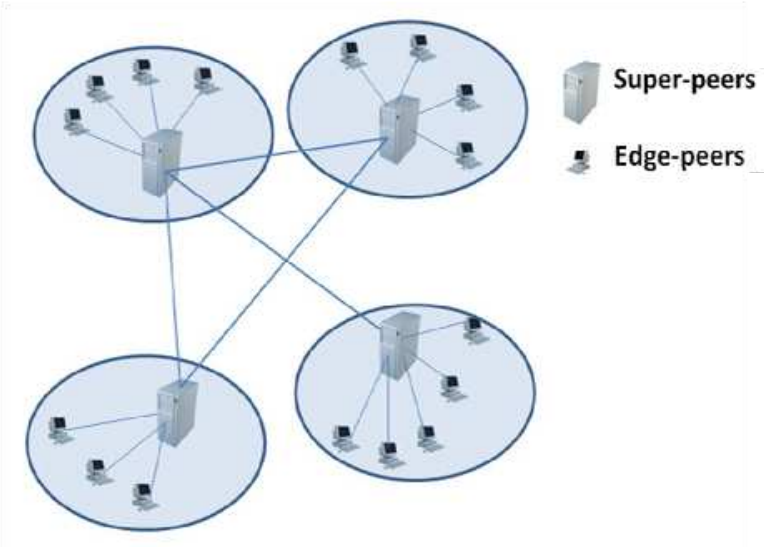

Figure 1. VICTORY network architecture

\section{TECHNOLOGY USED 3.1 P2P Middleware}

The next-generation of $\mathrm{P} 2 \mathrm{P}$ technologies is promising to provide the necessary infrastructure facilitating a seamless distribution of data and sharing of computational resources that are needed when building distributed digital repositories. Additional challenges for the $\mathrm{P} 2 \mathrm{P}$-based framework emerge from

Permission to make digital or hard copies of all or part of this work for personal or classroom use is granted without fee provided that copies are not made or distributed for profit or commercial advantage and that copies bear this notice and the full citation on the first page. To copy otherwise, or republish, to post on servers or to redistribute to lists, requires prior specific permission and/or a fee.

Conference'04, Month 1-2, 2004, City, State, Country.

Copyright 2004 ACM 1-58113-000-0/00/0004 ...\$5.00. the fact that it will be transparently used by both fixed and mobile peers.

As it has been mentioned, a hybrid architecture, utilizing pure P2P as well as client-server approaches. Pure P2P protocols are used when communication with devices behind firewalls and NATs is needed, therefore they are mainly used for the implementation of sharing and downloading services of files that are stored on edge peer devices. Project VICTORY uses JXTA technology to build high-level services. We chose JXTA because a broadly accepted standard for establishing an overlay P2P network over an existing low-level networking infrastructure is still missing. The only attempt in that direction is the project JXTA which tries to standardize a set of generic low-level protocols and services on top of which diverse P2P applications can be built. Also, VICTORY proposes the concept of "islands", where VICTORY island groups' users share common interests (like automotive group or tourist group). Further, a VICTORY island may provide some specific services (collaboration), while other services (search and download) are common to whole VICTORY community

The search and other services (rendering, QoE, identity management, and so on) are all located on known public IP addresses. For the communication with this ordinary HTTP transfer, Apache Tomcat and Servlet technologies are used.

The integration of these truly innovative search mechanisms with P2P technology should result in a flexible, secure, highperformance distributed MultiPedia repository resulting in high precision and recall measures for retrieval.

\subsection{Search Engine}

Content-based search relies on automated low-level geometric characteristics. The existing 3D retrieval systems allow the user to insert a 3D model as query. Novel algorithms regarding the $3 \mathrm{D}$ content-based search and retrieval based on the Radon Transform [4], Spherical Trace Transform [5], Moments [6,7] and combination of geometry with topology [8] have already been introduced. However, this research field still remains open because of various reasons: Firstly, there are no methods yet which can be applied to all 3D objects regardless of their degenerations and digital format, neither are they invariant to objects affine transformations. Furthermore, there exist only a few methods that combine partial (2D/3D or sketches/3D) matching.

Context based search mechanisms will be based on highlevel features of MultiPedia objects, which will be mapped to low-level geometric features. Semantic information representing high-level features will be managed in a form of ontology. The use of ontology and the ability to reason over the concepts and relations stored in it are the key components of the next generation of semantic knowledge technologies.

\subsection{Application-level services}

\subsubsection{Visualization on mobile devices}


The visualization of 3D geometries on desktop devices is a well investigated task and it can be accomplished in different ways. OpenGL/DirectX based applications are able to take advantage from the hardware support of nowadays graphics cards. On the other hand, the visualization on poor resource devices such as mobile terminals is still a challenging task. Although most recent devices provide hardware implemented rendering pipelines, complex scenes are still out of the power capabilities of handheld and mobile terminals. The remote visualization paradigm can be successfully employed in these cases [9]. In Victory, rendering servers will be able to manage 3D geometries (the most common format files will be supported: DXF, VRML, 3DS, and so on) and can accept connections from remote clients. A rendering server computes the scene simultaneously at different image resolutions (in order to support different categories of client devices); a connected client receives only a data representation (e.g. a flow of still images or a video stream) and sent back to the rendering server a set of commands needed to investigate the scene. The application running at the rendering server side will be based on OpenGL/OpenSG libraries in order to support high performance collaborative visualization session.

VICTORY will be also able to support collaborative visualization sessions; users can be concurrently display a shared Multipedia object both using desktop and mobile devices.

Moreover, a novel methodology for the remote control of application will be introduced by VICTORY [10]. The proposed solution is articulated in five steps: I) the remote application GUI is automatically analyzed and classified through image processing techniques, II) a description is generated using an extensible language derived from XML, III) the interface description is reloaded on the client side by a specific application and all the graphics elements previously classified (buttons, menus, check boxes, text fields, etc.) can be placed by the user in a personalized way, IV) any interaction with the client interface will produce an interaction on the corresponding graphics element in the remote application interface, V) finally, the work area (or one or more portion(s) of it, depending on user needs) is managed as a flow of still pictures delivered by the server to the mobile client.

\subsubsection{Relevance feedback}

In modern 3D databases, there is an imperative need for accurate retrieval of objects that are semantically similar to user queries, in order to reuse 3D objects and to exploit knowledge relative to them. Most current methods are making advances in geometric feature extraction in order to improve the retrieval accuracy. Clearly, if the discriminative power of such features was omnipotent, there would be no need of other techniques for improving retrieval or classification of $3 \mathrm{D}$ objects as a preprocessing step. It is also clear though, that no feature extraction scheme is perfect and thus, additional techniques are necessary. The most powerful technique in improving retrieval adequacy, apart from devising better low-level descriptors, is the use of Relevance Feedback (RF).

In RF schemes, the user becomes an active part of the retrieval system. Firstly, s/he supplies the system with a query. The system responses with a list of similar objects. The user gives relevance scores to a number of objects of the list according to his/her own judgement. Then, these scores are fed back to the system, which refines the search and responds with a new list that contains objects which are more similar to the user query.

In this context new RF methods developed in VICTORY able to combine both low-level and semantic features, in one single formula, in order to improve the retrieval results. Furthermore, the problem of pure semantic retrieval is a current research topic that has, still, been minimally explored and was addressed with an innovative RF method which provides promising results [11].

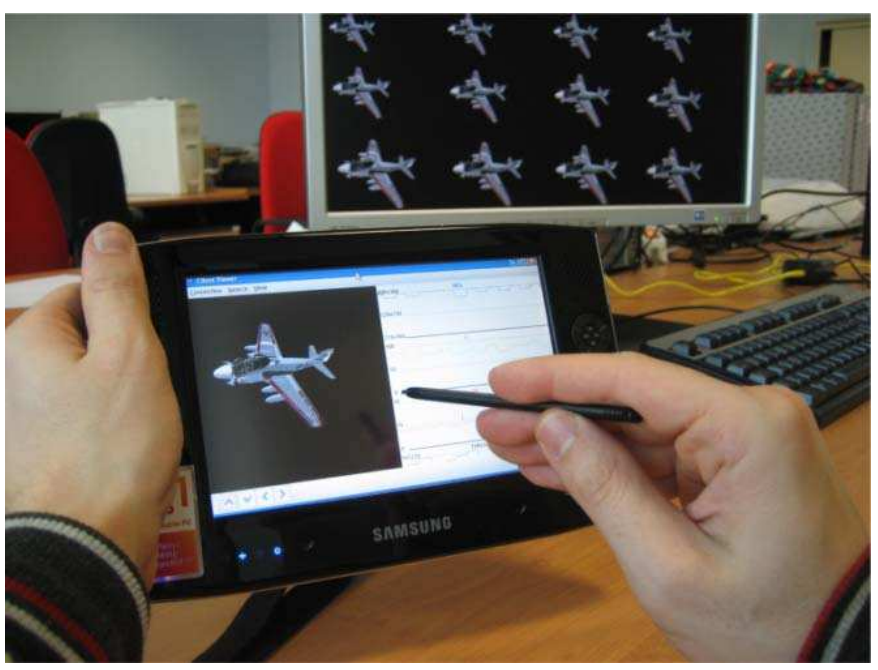

Figure 2. Visualization of 3D objects in mobile devices

\subsubsection{Digital Rights Management}

As mentioned earlier, a vast amount of multimedia objects is nowadays publicly available. The need for proper protection of their digital content and the associated rights is considered of great importance. A Digital Rights Management System (DRM) provides to the creator and the owners of digital content to limit the usage of digital media. Within VICTORY, DRM provides protection and control over the rights of the MultiPedia objects. It supplies the necessary functionality for the end users to digitally manage the rights of content as well as a secure and resilient way to distribute content and rights to the end users.

The DRM module that is implemented within the VICTORY context consists of different subsystems: the content producer, the content issuer, the rights issuer, the content consumer, the rendering engine, the identity management and watermarking (the last two are external to DRM). DRM system permits to the end users, consumers of Victory infrastructure, to access content through the Content providers, obtain the relevant Rights objects from an Issuer and consequently consume the content into a DRM enabled player. The Rendering Engine module is not only in charge to support the user for remote visualization sessions but also capable of enforcing the DRM constraints in cases where the MultiPedia object is DRM protected. Finally, the identity management subsystem provides the requisite mechanisms for the proper identification of the users and the entities involved in the DRM system. 


\subsubsection{Quality of Experience (QoE) manager}

An integrated and distributed QoE manager will be able to dynamically accomplish user needs during remotely rendering supported visualization sessions. In particular, a part of the QoE manager will run on rendering servers and a second part of (mobile) client devices. The QoE manager will be able to "tune": frame resolution, image quality, and frame rate according to the available throughput (the throughput is basically related to the bandwidth and to the decoding capability of the client device); moreover, in order to fulfil user requirements, the QoE manager will allow the user to specify a priority order for these parameters (Figure 2 shows an example of remote rendering session on an Ultra PC Samsung Q1; on the background the remote rendering console is visible).

The QoE involved in a remote visualization session is basically related to: frame rate, image resolution, quality, and available bandwidth. The frame rate affects the degree of interactivity and it is strongly dependent on the application (highly interactive applications such as video games need high frame rates), while image resolution can be a critical issue if small details of geometry have to be appreciated. The quality is often a subjective perception, but it can be quantitatively controlled in lossy compression schemes such as Jpeg. At the application level, the bandwidth can be periodically measured but, in general, it is not realistic to make any assumption about the available QoS. The QoE manager of Victory aims at concurrently considering the above parameters together with user preferences in order to maximize the his/her satisfaction. For this, a so called QoE graph providing a formal representation of the remote visualization parameters is designed, and a QoE manager capable of exploiting graph based information to tailor system behavior to user needs is developed. In this graph, each node represents a possible configuration for the characteristics of the image flow sent to the client device by the remote rendering server. Each particular configuration is expressed by a pair of values, namely resolution $\mathrm{R}$ and quality $\mathrm{Q}$ (where the term quality is referred to the Jpeg encoding). The edges of the graph represent possible transitions between nodes, and are determined according to a metric that is capable of taking into account the current available bandwidth as well as with user requirements. In particular, user requirements are characterized in terms of desired frame rate and priorities assigned to each controllable parameter that is frame rate, resolution, and quality. The proposed methodology dynamically builds and reconfigures the graph by periodically measuring the available bandwidth. Then, the graph is travelled in order to reach the node (that is, the configuration) that both provides the best bandwidth occupation and satisfies user requirements. A set of transition rules guarantees a smooth passage among adjacent nodes that is therefore carried out without noticeable changes in the visualization. After a rapid transitory, the QoE manager is able to identify the optimal configuration that is continuously adjusted during the whole visualization session.

\section{CONCLUSIONS}

The VICTORY project provides new insight into the nature of next generation audiovisual content search engines. It is eveloping novel architectures for access and delivery of multimedia content over an interoperable environment, which comprises standard and mobile P2P networks. It will foster innovative research on collaborative systems and develop new mechanisms for interaction and sharing of audiovisual content among users.ACKNOWLEDGMENTS

This work was supported by the EC Project VICTORY

\section{REFERENCES}

[1] 3-D That Could Transform The Web, News Flash Technology, Business Week, August 10th, 2000, http://www.businessweek.com/bwdaily/dnflash/aug2000/ nf20000810_036.htm.

[2] IST VICTORY project page, http://www.victory-eu.org

[3] Jxta v2.0 protocols specification. http://spec.jxta.org/nonav/v1.0/docbook/JXTAProtocols.html

[4] P. Daras, D. Zarpalas, D. Tzovaras and M. G. Strintzis, "Efficient 3D Model Search and Retrieval Using Generalized 3D Radon Transforms", IEEE Trans. on Multimedia Forman, G. 2003. An extensive empirical study of feature selection

[5] P. Daras, D. Zarpalas, D. Tzovaras and M.G. Strintzis: "3D Model Search and Retrieval Based on the Spherical Trace Transform", IEEE International Workshop on Multimedia Signal Processing (MMSP 2004), Siena, Italy, October 2004.

[6] D. Zarpalas, P. Daras, D. Tzovaras and M. G. Strintzis, “3D Content-based Search based on Fourier Transforms", In Proceedings of the 6th International Workshop on Image Analysis for Multimedia Interactive Services (WIAMIS 2005), Montreux, Switzerland, April 13-15, 2005.

[7] P. Daras, D. Zarpalas, D. Tzovaras and M.G. Strintzis, "3D Model Search and Retrieval Based on the Spherical Trace Transform", In Proceeding of the IEEE International Workshop on Multimedia Signal Processing (MMSP 2004), Siena, Italy, Sept 29 - Oct 1, 2004.

[8] A. Mademlis, P. Daras, A. Axenopoulos, D. Tzovaras, and M. G. Strintzis, "Combining Topological and Geometrical Features for Global and Partial 3D Shape Retrieval", IEEE Transactions on Multimedia, Volume 10, Issue 5, August 2008

[9] F. Lamberti and A. Sanna, "A streaming-based solution for remote visualization of 3D graphics on mobile devices", IEEE Transactions on Visualization and Computer Graphics Vol. 13, No. 2, pp. 247-260, 2007, ISSN: 1077-2626.

[10] F. Lamberti and A. Sanna, "Extensible GUIs for Remote Application Control on Mobile Devices", IEEE Computer Graphics and Applications, July-August 2008, ISSN: 02721716.

[11] E. Onasoglou and P. Daras: "Semantic Force Relevance Feedback, Content-Free 3D Object Retrieval and Annotation Propagation: Bridging the Gap and Beyond", SPRINGER Multimedia Tools and Applications Journal (MTAP), Special Issue on Multimedia Semantics, DOI 10.1007/s11042-0080216-1, July 2008 